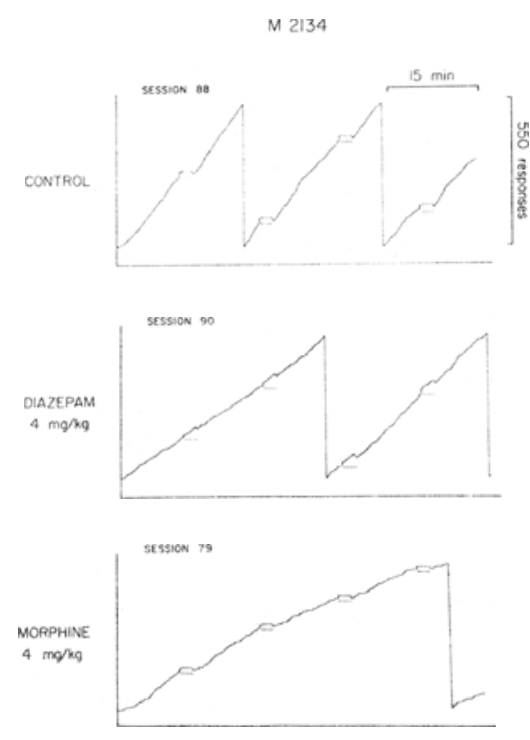

Fig. 2. Sample cumulative response records illustrating the effects of morphine and diazepam in one monkey. Each presentation of the preshock stimulus is indicated by an offset of the recorder pen and a horizontal line.

long-term stability, were eventually obtained with the following parameters: a preshock stimulus of $2 \frac{1}{2} \mathrm{~min}$ duration presented four times during each session at regular 12-min intervals, the first coming $12 \mathrm{~min}$ after the beginning of the session; a current intensity of $10 \mathrm{~mA}$; and a shock duration of $5 \mathrm{sec}$. These parameters were in effect during the period of drug testing.

Morphine produced only a dose-related depression of lever responding in both components of the schedule (Fig. 1). Diazepam, a drug reported to increase responding suppressed by a preshock stimulus in rats (Xhenseval, 1964), was tested in the same monkeys. In contrast to morphine, diazepam $(4.0 \mathrm{mg} / \mathrm{kg})$ produced a significant increase in responding during the preshock stimulus.

Sample cumulative response records of one monkey are shown in Fig. 2. The increase in lever responding during the preshock stimulus produced by diazepam can be compared with the general depressant action on all responding exerted by morphine.

\section{DISCUSSION}

The findings that morphine restored behavior suppressed by preshock stimuli in rats (Hill et al, 1954; Maxwell et al, 1961) prompted Hill et al $(1954,1966)$ to suggest that morphine is effective in relieving pain-anticipatory anxiety, and that the analgesic action of the drug is partially a reflection of anxiety reduction. However, in the present study, morphine failed to reduce conditioned suppression of behavior in monkeys and produced only a dose-related depression of operant responding in both components of a VI schedule. A similar depression of behavior by morphine in rats was reported by Lavener (1963). Hill et al (1966, 1967) suggested that the discrepancy between their findings and those of Lauener (1963) was possibly related to differences in such parameter values as the intensity and frequency of presentations of the preshock stimulus.

The findings of the present study, although based on a small number of observations, indicate that the reduction of conditioned suppression by morphine is dependent upon procedural details and perhaps also upon species. Thus, the effect of morphine in reducing conditioned suppression lacks sufficient generality to lend support to the view that the analgesic effect of morphine is related to a reduction in anxiety associated with the anticipation of pain. Furthermore, the restoration of suppressed behavior is not exclusive for drugs with pain-relieving properties, as demonstrated by the fact that conditioned suppression was attenuated by diazepam, a drug devoid of significant analgesic activity (Zbinden \& Randall, 1967).

\section{REFERENCES}

ESTES, W. K., \& SKINNER, B. G. Some quantitative properties of anxiety. Joumal of Experimental Psychology, 1941, 29, 390-400. HILL, H. E., BELLEVILLE, R. E., \& WIKLER, A. Reduction of pain-conditioned anxiety by analgesic doses of morphine in rats. Proceedings of the Society for Experimental \& Biological Medicine, 1954, 86, 881-884.
HILL, H. E., BELLEVILLE, R. E., PESCOR, F. T., \& WKLER, A. Comparative effects of methadone, meperidine and morphine on conditioned suppression. Archives Internationales de Pharmacodynamie et de Thérapie, 1966, 163, 341-352.

HILL, H. E., BELL, E. C., \& WIKLER, A. Reduction of conditioned suppression: Actions of morphine compared with those of amphetamine, pentobarbital, nalorphine, cocaine, LSD-25 and chlorpromazine. Archives Internationales de Pharmacodynamic et de Thérapie, 1967, 165, 212-226.

LAUENER, H. Conditioned suppression in rats and the effect of pharmacological agents thereon. Psychopharmacologia, 1963, 4, 311-325.

MAXWELL, D. R., PALMER, H. T., \& RYALL, R.W. A comparison of the analgesic and some other properties of methotrimeprazine and morphine. Archives Internationales de Pharmacodynamie et de Thérapie, 1961, 132, 60-73.

SIDMAN, M. Normal sources of pathological behavior. Science, 1960, 132, 61-68.

XHENSEVAL, B. Action comparée de méprobamate et du diazepam sur le comportement conditionné chez le rat. Joumal de Physiologie, 1964, 56, 465-466.

ZBINDEN, G., \& RANDALL, L. 0. Pharmacology of benzodiazepines: Laboratory and clinical correlations. In S. Garattini and P. A. Shore (Eds.), Advances in pharmacology. New York: Academic Press, 1967. Pp. 213-291.

\section{Supported by USPHS Grant} R10-MH-12084-02.

2. Present address: Department of Pharmacology, Emory University, Atlanta, Ga. 30322.

3. The diazepam used in this study was generously supplied by Mr. Rostom Tandourjian of Hoffman-LaRoche, Inc. Expert technical assistance was provided by Messrs. Fred Adams and Clifton Fly.

\title{
Effects of sodium deficiency on sodium saccharin drinking 1
}

\section{F. SAMUEL BAUER, ${ }^{2}$ University Illinois, Urbana, Ill. 61801}

This experiment attempts to test Warren \& Warren's (1966) hypothesis that the persistence of intake of nonnutritive sodium saccharin is due in part to the sodium content of the solution. The intakes of sodium saccharin, sodium chloride, and deionized water by sodium-sufficient and by sodium-deficient rats were compared, using a single-stimulus technique. The results contradict Warren and Warren's findings, leading to the conclusion that sodium does not play an important role in saccharin preference.

In reply to Warren \& Warren (1966), who suggested that the persistence of soluble saccharin intake as compared to that of insoluble saccharin was due in part to the sodium content of the soluble saccharin, Valenstein (1966) showed that the difference in the intakes of these substances was probably due simply to differential taste 
preferences. Valenstein pointed out that equimolar solutions of the two types of saccharin are easily distinguished by human Os; however, he concluded that he could not rule out the possibility that the metabolic effect of sodium in some way influenced the taste preferences.

The present study compares the intakes of sodium saccharin, equimolar sodium chloride, and water of both sodium-deficient and sodium-sufficient rats in order to assess the importance of sodium in the preference for sodium saccharin.

\section{METHOD}

Thirty-six 90-day-old female Holtzman albino rats, weighing from 240 to $260 \mathrm{~g}$ at the beginning of the experiment, were housed individually on wire-mesh floors. Food was delivered in metal food cups (Wahmann 306/B), equipped with baffles to reduce spillage. Fluids were presented in inverted $100-\mathrm{ml}$ graduated cylinders with aluminum drinking tubes.

The Ss were randomly divided into six groups. Three groups were maintained on a sodium-deficient diet (Hartroft \& Eisenstein, 1957) purchased from General Biochemicals Co. (Chagrin Falls, Ohio), and the other three groups were maintained on a control (sodium-sufficient) diet of the same formula, but with normal amounts of sodium replaced. Food was offered ad lib, as was deionized water. After 21 days, tail blood samples were taken from three $S s$ in each of the dietary conditions. The blood was collected in heparanized pipettes, flame-sealed, centrifuged, and frozen, and then the thawed samples were analyzed for plasma sodium on the flame photometer.
The plasma sodiums were pooled over dietary group, and a t test for the difference in the means was run. This test was significant at the .01 level $(t=6.24, d f=4)$, hence verifying a difference in sodium levels and indicating the start of the preference-testing phase of the experiment.

The testing phase used a single-bottle preference test. One group from each of the dietary conditions (six Ss per group) was given an $8.2 \times 10^{-3}$ molar $(0.198 \%)$ sodium saccharin solution, an $8.2 \times 10^{-3}$ molar ( $0.047 \%)$ sodium chloride solution, or deionized water. All solutions were prepared and changed daily.

The solutions were presented for 7 consecutive days. On the first and last days, blood samples were taken from all Ss in the manner previously described. During these test days, food intake, fluid intake, and body weight were measured daily. The animals were fed the same diet in this phase as during the earlier phase.

\section{RESULTS AND DISCUSSION}

Figure 1 summarizes the mean daily intakes for each of the six experimental groups for each test day. A 2 by 3 by 7 analysis of variance with repeated measures on the days factor was performed on these data. The significant factors were Test Solution $(\mathrm{F}=57.0, \mathrm{df}=2 / 30)$, Days $(F=25.4, d f=6 / 180)$, Test Solution by Days interaction $(F=6.40, \mathrm{df}=12 / 180)$, Diet by Days interaction ( $F=5.8$, $\mathrm{df}=6 / 180$ ), and the three-way interaction $(F=3.2, \mathrm{df}=12 / 180)$. Each of these factors was significant at the .01 level.

Figure 1 shows that both water and sodium chloride intakes were similar over

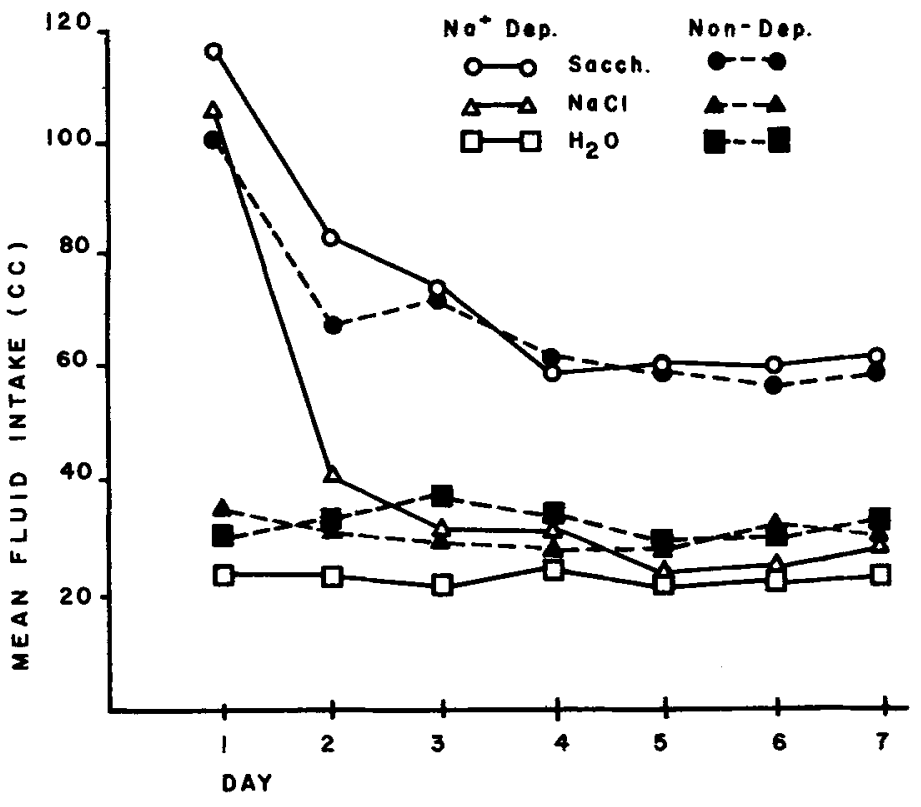

Figure 1 both dietary conditions and test days. Saccharin intake, however, for both dietary conditions is quite high, although it shows some decline over the test days, probably accounting for the significant Days effect and the Solution by Days interaction.

The blood tests verified the sodium deficiency at the beginning of the test period, and also showed that this deficiency was not made up at the end of the period. At the beginning, the plasma sodium means $(\mathrm{mEq} / 1)$ of the two dietary conditions differed significantly $\left(M_{\text {suff }}=142.8\right.$, $\mathrm{M}_{\mathrm{dep}}=104.2, \mathrm{t}=22.5, \mathrm{df}=34$ ) at the .01 level, while after the test, the means were still significantly different at the same level $\mathrm{M}_{\mathrm{suff}}=141.7, \quad \mathrm{M}_{\mathrm{dep}}=120.1, \quad \mathrm{t}=14.2$, df = 34).

The high initial intake of sodium chloride by the sodium-deficient animals seems to be the primary source of the Diet by Days interaction. This phenomenon provides a problem of interpretation, especially since the data show that sodium deficits were not made up. One possible explanation for this high intake would be a lowered threshold for sodium chloride drinking due to the sodium deficiency, as suggested by Richter (1939) for adrenalectomized rats.

The results indicate that sodium saccharin intake is not modified by sodium need. Thus, the metabolic consequence of the small amounts of sodium in the sodium saccharin solutions is negligible in determining the preference for these solutions. This interpretation is verified by comparing the levels of intake of the sodium chloride and sodium saccharin that are matched for sodium ion concentration. These results are in agreement with the results of Valenstein (1966), and are in partial opposition to the findings of Warren \& Warren (1966).

\section{REFERENCES}

HARTROFT, $P$. M., \& EISENSTEIN, A B. Alterations in the adrenal cortex of the rat induced by sodium deficiency: Correlation of histologic changes with steroid hormone secretion. Endocrinology, 1957, 60, 641-651.

RICHTER, C. P. Salt taste threshold for normal and adrenalectomized rats. Endocrinology, $1939,24,367-371$.

VALENSTEIN, E. S. The significance of sodium in the preference for saccharin solutions. Psychonomic Science, 1966, 5, 341-342.

WARREN, R. P., \& WARREN, R. M. Soluble saccharin preference: A nutritive basis for persistence. Nature, 1966, 210, 310-311. NOTES

1. This study is based on a thesis submitted to the Graduate College of the University of Illinois in partial fulfillment of the requirements for the MA degree in psychology. The author is grateful to Prof. L. I. O'Kelly, chairman of the thesis committee, to Prof. G. R. Grice who served as second reader, and to Ellen $R$. Bauer for her editorial assistance.

2. Now at: Department of Psychology, The College of William and Mary, Williamsburg, Va. 23185 